\title{
Effect of Minor Titanium Addition on Copper/Diamond Composites Prepared by Hot Forging
}

\author{
Fei Yang, ${ }^{1,2}$ Wei Sun, ${ }^{1}$ Ajit Singh, ${ }^{1}$ Leandro Bolzoni ${ }^{1}$ \\ 1.-Waikato Centre for Advanced Materials, School of Engineering, The University of Waikato, Hamilton 3240, New Zealand. \\ 2.—e-mail: fei.yang@waikato.ac.nz; fyang0204@hotmail.com
}

Copper/diamond composites have great potential to lead the next generation of advanced heat sink materials for using in high-power electronic devices and high density integrated circuits, due to its potential excellent properties of high thermal conductivity and close thermal expansion to the chip materials (e.g. Si, InP, GaAs). However, the poor wettability between copper and diamond presents a challenge for synthesising copper/diamond composites with effective metallurgical bonding and satisfied thermal performance. In this paper, copper/diamond composites were successfully prepared by hot forging of elemental copper and artificial diamond powders with small amounts ( 0 vol.\%, 3 vol.\% and 5 vol.\%) of titanium additives. Microstructure observation and mechanical tests showed that, adding minor titanium additions in the copper/diamond composite resulted in fewer cracks in the composite's microstructure and significantly improved the bonding between the copper and. The strongest bonding strength was achieved for the copper/diamond composite with 3 vol.\% titanium addition, and the possible reasons were discussed.

\section{INTRODUCTION}

With developing the electronic devices, the traditional electronic packaging materials are facing a great challenge ${ }^{1-3}$ as the heat fluxes in the electronic devices has dramatically increased..$^{3-5}$ Copper/diamond composites have attracted extensive attention due to their potentially high thermal conductivity and thermal expansion coefficients similar to those existing chip materials. However, preparing copper/diamond composite materials with satisfactory thermal conductivity and expansion has proven difficult due to the poor wettability between copper and diamond, which results in weak interfacial bonding.

Recent research suggested that the thermal conductivity of copper/diamond composites could be improved by adding minor elements, such as $\mathrm{Cr}^{6-7} \mathrm{Ti}^{8-9}$ and $\mathrm{B} .{ }^{10}$ The most common manufacturing technologies used to produce these composites were vacuum hot pressing, ${ }^{11}$ high temperature high pressure (HTHP) ${ }^{12}$ gas pressure infiltration, ${ }^{13}$ pulse plasma sintering ${ }^{6}$ and spark plasma sintering, ${ }^{14}$ all of which require highly specialised equipment. Consequently, any such composites are prohibitively expensive materials, which impedes their commercial applications. Impurities such as oxides and intermetallic compounds, ${ }^{8}$ can also form during the synthesis process, and may influence the heat flow, decreasing the composites' thermal properties. Hot forging is a potential low-cost manufacturing method for producing solid parts from powder, as the fast heating has the potential to limit or even eliminate the formation of any impurities. In this study, we explored the fabrication of copper/diamond composites from elemental powder mixtures using hot forging combined with induction heating, and then 
investigated the microstructure, phase constitution and interface bonding of the resulting copper/diamond composite.

\section{EXPERIMENTAL PROCEDURE}

Copper-55vol.\%diamond-xTi ( $\mathrm{x}=0,3$ and 5 vol.\%) composites (copper/diamond composite) were produced by hot forging of a powder mixture of copper, diamond, and hydride-dehydride (HDH) titanium powders. The copper powder was produced by rapid-solidification and had a purity of $99.9 \%$ and particle size of smaller than $90 \mu \mathrm{m}$. The HDH titanium powder had a purity of higher than $99.6 \%$ and particle size of smaller than $75 \mu \mathrm{m}$ (200 mesh). The SCMD-AA synthetic diamond powder used was supplied by Henan Yuxing Sino-crystal Micro-diamond Co. Ltd, PR China, and its particle size was approximately $50-60 \mu \mathrm{m}$. The copper, diamond and titanium powders were first mixed for $24 \mathrm{~h}$ using a planetary ball mill at a rotating speed of $100 \mathrm{rpm}$; the ball-to-powder weight ratio for mixing was $2: 1$, and the ball for mixing was made of stainless steel and had a diameter of approximately $6 \mathrm{~mm}$. The powder mixture was then hot forged at $1000-1050^{\circ} \mathrm{C}$ under a maximum pressure of $400 \mathrm{MPa}$ in an argon atmosphere with oxygen content below $200 \mathrm{ppm}$ to produce a pancake. The overall deformation was about $74 \%$.

Scanning electron microscopy (SEM) (HITACHI S4700) and X-ray diffraction (XRD) (Philips X-pert $\mathrm{X}$-ray diffraction system, $\mathrm{Cu} \mathrm{K} \alpha$ radiation and a graphite monochromator) were used for microstructure observation and phase analysis. The microstructure observation sample was taken from the direction perpendicular to the normal direction (ND) of the as-forged pancake. Thermo Noran EDS, which was attached to the HITACHI S4700 and had an accelerating voltage of $20 \mathrm{kV}$, was used to measure the chemical composition and element distribution. The bulk density of the forged copper/diamond composites was measured using the Archimedes method.

Three-point bending tests were performed at room temperature using an Instron (INSTRON 4204) universal testing machine to determine the transverse rupture strength (TRS) of the copper/diamond composites. During the test, the loading direction was parallel to the ND, the test sample had a dimension of $3 \mathrm{~mm} \times 4 \mathrm{~mm} \times 30 \mathrm{~mm}$, and the span and strain rate were $20 \mathrm{~mm}$ and $3 \times 10^{-3} \mathrm{~s}^{-1}$, respectively. The fracture surfaces of broken samples were examined using SEM.

\section{RESULTS AND DISCUSSION}

\section{Phase constitution of the as-forged copper/diamond composites}

XRD patterns of the as-forged copper/diamond composites are shown in Fig. 1, which shows TiC peaks in both of the $3 \mathrm{vol} \% \mathrm{Ti}$ - and $5 \mathrm{vol} \% \mathrm{Ti}$ - copper/diamond composites. These peaks suggest that the chemical reaction between the titanium $(\mathrm{Ti})$ and diamond $(\mathrm{C})$ forms $\mathrm{TiC}$ phase during the process of heating and forging the powder mixture. The intensity of the $\mathrm{TiC}$ phase in the 5 vol.\% Ticopper/diamond composite is higher than that in the 3 vol.\% Ti-copper/diamond composite, and this is attributed to that $\mathrm{Ti}$ content is higher in the former composite. The formation of $\mathrm{TiC}$ between diamond and copper is expected to improve the adhesion of copper to diamond particles and enhance the bonding strength between copper and diamond.

$\mathrm{Ti}-\mathrm{Cu}$ intermetallic compounds, as reported to form in the copper/titanium-coated diamond composites, ${ }^{8}$ were not detected in the XRD patterns of the studied copper/diamond composites. We posit that this is mainly because the Ti powder is homogeneously distributed in the copper matrix after mixing for $24 \mathrm{~h}$. According to the phase diagram of $\mathrm{Ti}-\mathrm{Cu},{ }^{15}$ copper-5 vol.\% $\mathrm{Ti}$ is confined to single $\mathrm{Cu}$ region 
when the temperature is in the range of $1000-1050^{\circ} \mathrm{C}$. Although some Ti-Cu may have formed in minute quantities, no relative peaks were observed in the XRD patterns. Thus, Ti is more likely to form solid solution in $\mathrm{Cu}$ matrix rather than forming Ti-Cu intermetallic compounds. For the copper/titanium-coated diamond composites, the titanium coated the surface of diamond particles, and its concentration was higher in the localised area between the diamond and copper matrix. As a result, Ti-Cu intermetallic compounds formed easily during hot processing. In this case, $\mathrm{Ti}$ cannot contribute to improving the wettability between copper and diamond.
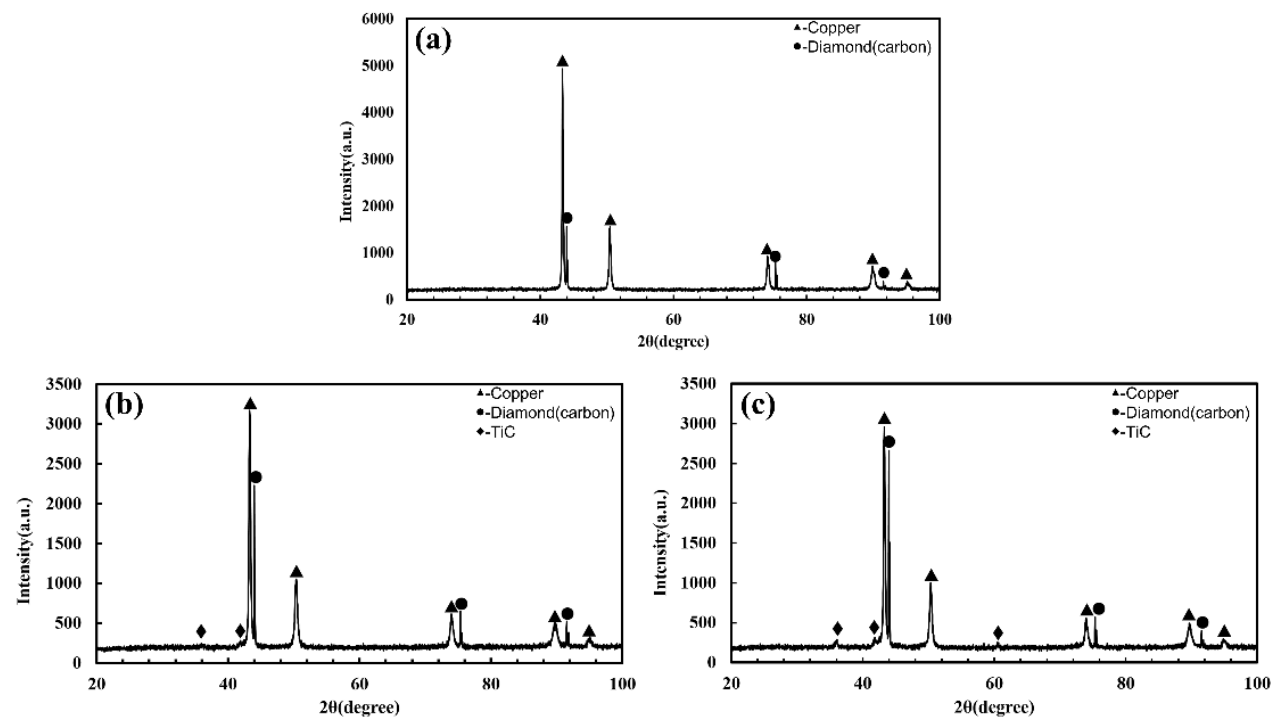

Fig. 1. XRD patterns of the as-forged copper/diamond composites with different volume fractions of titanium (a) 0 vol.\% Ti, (b) 3 vol.\% Ti, and (c) 5 vol.\% Ti.

\section{Microstructure of the copper/diamond composites}

Fig. 2 shows the SEM images of the as-mixed powder mixture of diamond, copper and titanium powders and the as-forged copper/diamond-3 vol.\% Ti composite after grinding and polishing. It can be seen that the diamond, copper and titanium powders are homogeneously mixed and the diamond powder was not damaged during the mixing progress (Fig.2a). Fig.2b shows that the diamond particles are homogeneously distributed in the copper matrix, with no obvious voids and/or gaps between the diamond particles and the copper matrix. The interface layers between some diamond particles and the copper matrix are visible (Fig.2c), suggesting that the diamond and copper are well bonded. The relative densities for copper/diamond composites with different amount of titanium additions are $96.72 \%$ for the 0 vol.\% Ti-composite, $97.78 \%$ for the 3 vol. $\%$ Ti-composite and $97 \%$ for the 5 vol. $\%$ Ti-composite, respectively. Thus, hot forging appears to facilitate the bonding of the diamond particles with the copper matrix.
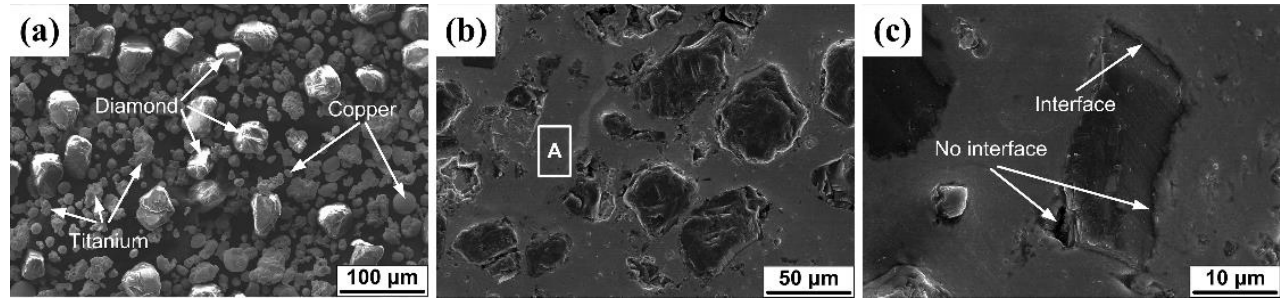
Fig. 2. SEM images of (a) the powder mixture of diamond, copper and titanium; and (b) and (c) the as-forged copper/diamond-3 vol.\% Ti composite.

Fig. 3 shows the high-magnification SEM images and EDS mapping and line-scanning analysis of the diamond, titanium, and copper elements at the vicinity of the interface between the diamond particles and the copper matrix. In EDS mapping, diamond is yellow colour, titanium is blue, and copper is purple. A large gap can be observed between the diamond and the copper in the 0 vol.\% Ti- copper/diamond composite (Fig.3a), which indicates weak bonding. ${ }^{16}$ For the 3 vol. $\%$ Ti- and 5 vol.\% Ti- copper/diamond composites, interface layers are visible and the results of EDS mapping and line-scaning analysis clearly show that the interfacial layer between the copper and diamond is rich in diamond and titanium. Combined with the XRD results (Fig.1b and c), this suggests that the TiC layer has formed between the copper and the diamond, with a thickness of about $1 \mu \mathrm{m}$. This TiC layer helps to reduce the gap between the copper and diamond particles, improving the composites' relative density and the adhesive force between the copper and diamond. Thus, it is clear that titanium addition has a significant effect on interfacial bonding and densification of the copper/diamond composites.

The interface microstructure varied significantly with the changes of diamond particle surfaces, as shown in Fig. 2b. The dissolution of carbon into minor elements is an important first step in the formation of interfacial carbides, ${ }^{5,17}$ and the dissolution ability is varied in different diamond surfaces due to the different atomic arrangement. It reported that $\mathrm{C}$ atoms were two-fold bonded in $\{100\}$ surface and threefold bonded in $\{111\}$ surface, leading to that the dissolution of carbon into minor elements on $\{100\}$ surface was easier than on $\{111\}, 5,17$ Thus, it can predicate that the planes on which TiC are formed would be the $\{100\}$ planes of diamond in the studied copper/diamond composites.
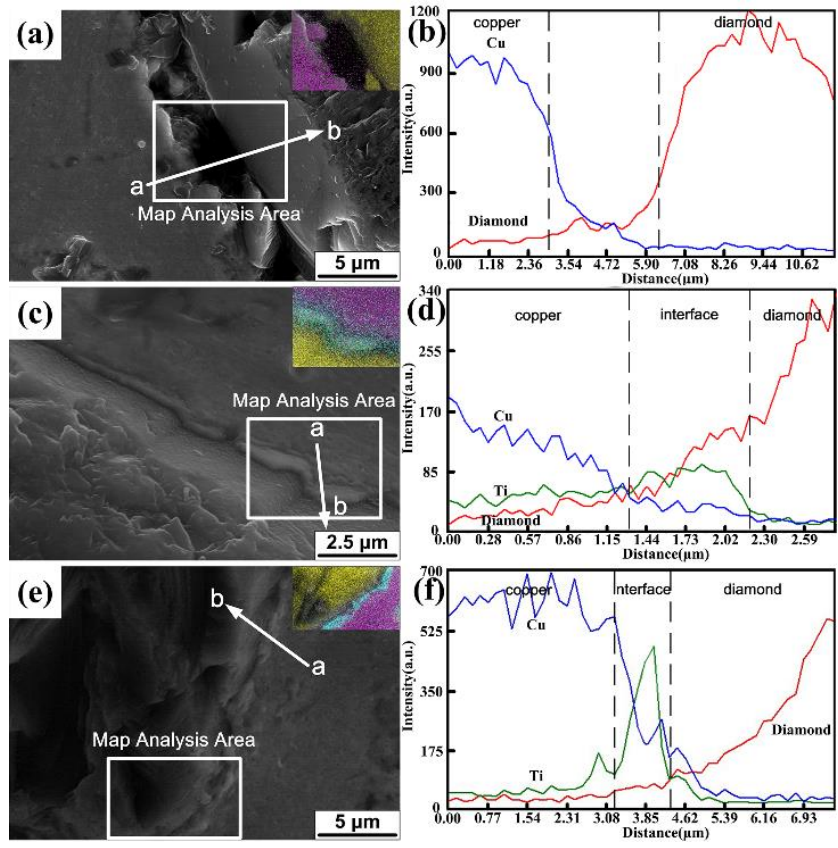

Fig. 3. Microstructures, EDS mapping and line-scanning analysis of the interface between the copper and diamond particles of the as-forged copper/diamond composites: (a) (c) (e) microstructures and mapping analysis of copper/diamond composite with 0 vol.\% Ti, 3 vol.\% Ti and 5 vol.\% Ti, respectively; and (b), (d) and (f) show the line-scanning analysis from point a to point $b$ in (a), (c) and (e), respectively. In EDS mapping: Diamond (C) is yellow, Ti is blue, and $\mathrm{Cu}$ is purple. 


\section{The three-point bending test and the fractography of composites}

Fig. 4 shows the transverse rupture strength (TRS)-deflection curves of the three copper/diamond composites. The TRS values are 119.3MPa for the 0 vol.\% Ti-copper/diamond composite, 317.1MPa for the 3 vol.\% Ti-copper/diamond composite, and 290.8MPa for the 5 vol.\% Ti-copper/diamond composite, respectively. The TRS values for the 3vol.\% Ti- and 5vol\% Ti- copper/diamond composites are almost two times higher than that of the composite with no titanium addition. According to previous studies, ${ }^{18}$ the TRS for a diamond composite is governed by both of the bonding force between the diamond and the metal matrix and the bonding force of the metal matrix. Since the metal matrix and forging conditions for the three copper/diamond composites were identical, the different TRS values for the studied copper/diamond composites solely reflected changes in the diamond-copper matrix bonding. The higher the TRS, the stronger the copper-diamond bonding; thus, our results suggest that the interface bonding between copper and diamond can be increased by adding titanium in the copper/diamond composites. The solution of $\mathrm{Ti}$ in copper matrix may also enhance the TRS for the studied copper/diamond composites, however, it reported that the solution strengthening (without aging) was limited for $\mathrm{Cu}-\mathrm{Ti}$ alloy when the Ti content was less than $1.5 \mathrm{wt} . \% .{ }^{19}$ In this study, the EDS result shows that the Ti content in the copper matrix is about 1 wt.\% for the 3 vol.\% Ti-copper/diamond composite (Fig. 2b, area A). Thus, the formation of the $\mathrm{TiC}$ layer in the copper/diamond composites is the primary driver of the improved bending strength.

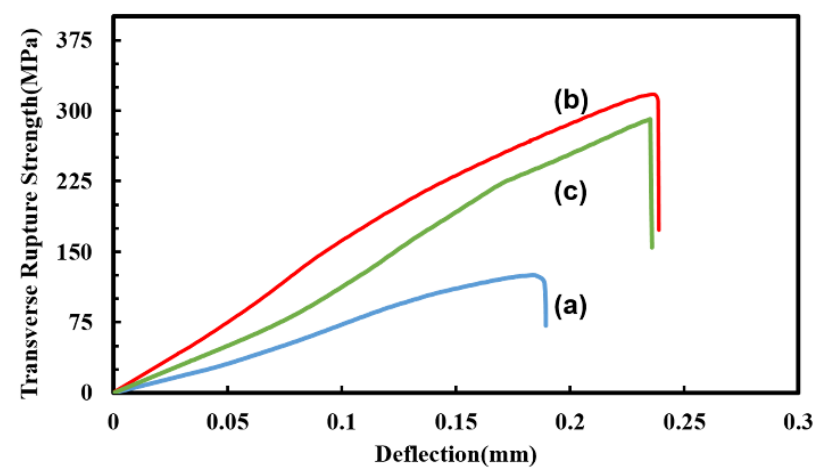

Fig. 4. Transverse rupture strength (TRS)-deflection curves for the copper/diamond composites with (a) 0 vol.\% Ti, (b) 3 vol.\% Ti, and (c) 5 vol.\% Ti.

Fig. 5 shows the fracture morphologies of the ruptured copper/diamond composites. The diamond particles protruded from the copper matrix, and clear gaps can be seen between the copper and diamond particles in the 0 vol.\% Ti-copper/diamond composite (Fig.5a). These gaps are less obvious in the 5 vol.\% Ti-copper/diamond composite (Fig. 5c) and not present in the 3 vol.\% Ti-copper/diamond composite (Fig. 5b). Furthermore, the transgranular fracture is present in the diamond particles for the 3 vol.\% Ticopper/diamond composite, but not in the copper/diamond composites with 0 and 5 vol.\% titanium additions. This difference further confirms that the interface bonding strength between the copper and diamond is higher for the 3 vol.\% Ti-copper/diamond composite .

Due to the poor wettability between copper and diamond, effective metallurgical bonding between copper and diamond requires the addition of other minor elements such as titanium. Thus, the interface bonding in the 0 vol.\% Ti- copper/diamond composite is poor, resulting in low TRS, and the diamond 
protrudes from the copper matrix in the fracture surface of the three-point test specimen. Adding small quantity of titanium allows TiC layer to form between the copper and diamond particles, which not only bonds the copper and diamond together, but also reduces the gaps between the copper and diamond particles in the resultant composite, leading to composites with higher relative densities. This increase in density is the main reason for the higher TRS values observed for the titanium-added copper/diamond composites, and the transgranular fracture surface observed in the 3 vol.\% Ti-copper/diamond composite. However, it is well known that carbides are very hard and brittle $;{ }^{20}$ thus, there is an optimal TiC amount to produce a better interface bonding between copper and diamond. The higher the titanium contents of the starting powder mixture, the greater the amount of $\mathrm{TiC}$ formed in the copper/diamond composites. Thus more $\mathrm{TiC}$ will be present in the $5 \mathrm{vol} . \%$ Ti-copper/diamond composite than that of the 3 vol.\% Ticopper/diamond composite. This difference may cause lower the TRS value observed for the copper/diamond composite with 5 vol.\% titanium addition, and we speculate that the thickness and morphology of the TiC layer between copper and diamond affects the bonding strength of the copper/diamond interface, further influencing the thermal conductivity of the copper/diamond composites. This aspects will be discussed in future papers. Thus, it can be concluded that, for the asforged copper/diamond composites, the optimum content for Ti addition is 3 vol.\% for improving the bonding strength of the copper/diamond composites.
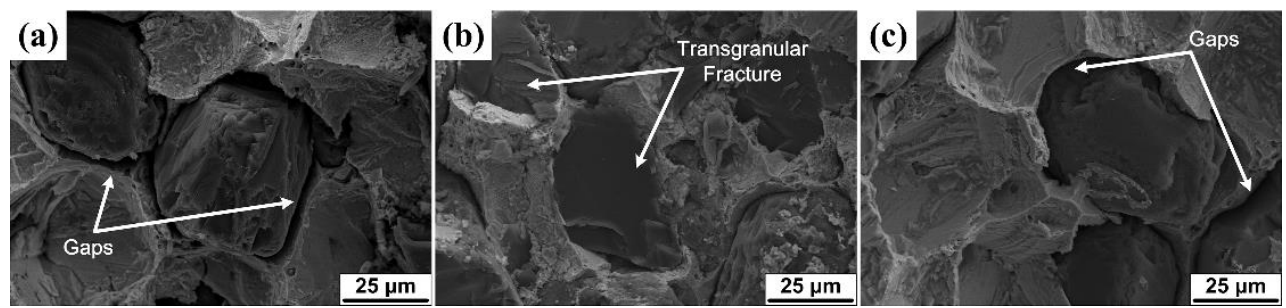

Fig. 5. The fracture surface of copper/diamond composites with (a) 0 vol.\% Ti, (b) 3 vol.\% Ti, (c), and 5 vol.\% Ti.

\section{CONCLUSION}

Copper/diamond composites with minor titanium addition can be successfully fabricated via the hot forging method, using copper, diamond and titanium powders. A clear TiC layer formed at the interface between the copper and diamond particles in the both 3 vol.\% Ti- and 5 vol\%Ti- copper/diamond composites. The $3 \mathrm{vol} \%$ Ti composite had the highest TRS value (317.1MPa compared to 119.3MPa for the $0 \mathrm{vol} \%$ Ti composite and $290.8 \mathrm{MPa}$ for the $5 \mathrm{vol} \%$ Ti composite) and a relative density of $97.78 \%$ relative density, reflecting the strong interfacial bonding achieved between the copper and diamond particles.

\section{ACKNOWLEDGEMENTS}

We gratefully acknowledged the funding from the US Air Force Asian Office of Aerospace R\&D (Grant No. FA2386-17-1-4025) and the University of Waikato, New Zealand (Grant No. 2017-SIFF759), that supported this work.

\section{REFERENCES}

1. T. Schubert, A. Brendel, K. Schmid, T. Koeck, L. Ciupinski, W. Zielinski, T. Weissgarber and B. Kieback, Compos. Part A: Appl. Sci. Manuf. 38, 2398 (2007).

2. J.M. Molina, M. Rheme, J. Carron and L. Weber, Scr. Mater. 58, 393 (2008). 
3. R. Arpon, J.M. Molina, R.A. Saravanan, C.G. Cordovilla, E. Louis and J. Narciso, Acta Mater. 51, 3145 (2003).

4. M. Schobel, H.P. Degischer, S. Vaucher, M. Hofmann and P. Cloetens, Acta Mater. 58, 6421 (2010).

5. P.W. Ruch, O. Beffort, S. Kleiner, L. Weber and P.J. Uggowitzer, Compos. Sci. Technol. 66, 2677 (2006).

6. J. Grzonka, M.J. Kruszewski, M. Rosiński, L. Ciupiński, A. Michalski and K.J. Kurzydłowski, Mater. Charact. 99, 188 (2015).

7. K. Chu, Z.F. Liu, C.C. Jia, H. Chen, X.B. Liang, W.J. Gao, W.H. Tian and H. Guo, J. Alloy. Compd. 490, 453 (2010).

8. Q.L. Che, X.K. Chen, Y.Q. Ji, Y.W. Li, L.X. Wang, S.Z. Cao, Y.G. Jiang and Z. Wang, Mater. Sci. Semicond. Process 30, 104 (2015).

9. Y. Zhang, H.L. Zhang, J.H. Wu and X.T. Wang, Scr. Mater. 65, 1097 (2011).

10. L. Weber and R. Tavangar, Scr. Mater. 57, 988 (2007).

11. W.P. Shen, W.J. Shao, Q.Y. Wang and M.L. Ma, Fusion Eng. Des. 85, 2237 (2010).

12. E.A. Ekimov, N.V. Suetin, A.F. Popovich and V.G. Ralchenko, Diam. Relat. Mater. 17, 838 (2008).

13. A.M. Abyzov, S.V. Kidalov and F.M. Shakhov, Appl. Therm. Eng. 48, 72 (2012).

14. S.B. Ren, X.Y. Shen, C.Y. Guo, N. Liu, J.B. Zang, X.B. He and X.H. Qu, Compos. Sci. Technol. 71, 1550 (2011).

15. J.L. Murray, Bull. Alloy Phase Diagr. 4, 81 (1983).

16. T. Schubert, B. Trindade, T. Weißgärber and B. Kieback, Mater. Sci. Eng. A 475, 39 (2008).

17. S. Kleiner, F.A. Khalid, P.W. Ruch, S. Meier and O. Beffort, Scr. Mater. 55, 291 (2006).

18. Y.S. Liao and S.Y. Luo, J. Mater. Sci. 28, 1245 (1993).

19. S. Nagarjuna, M. Srinivas, K. Balasubramanian and D. S. Sarma, Mater. Sci. Eng. A 259, 34 (1999).

20. K. Chu, C.C. Jia, H. Guo and W.S. Li, Mater. Des. 45, 36 (2013). 